\title{
Effects of Horseradish Oil and Eight Isothiocyanates Vapour Treatment on Postharvest Disease Control and Their Efficacy as Preservatives of Mature Green Tomato
}

\author{
Jing-jing Ren, ${ }^{1}$ Dan Zhang, ${ }^{1}$ Pu-xing Hou, ${ }^{1}$ and Hua $\mathrm{Wu}^{1,2, \dagger}$ \\ ${ }^{1}$ College of Plant Protection, Northwest A\&F University, Yangling, Shaanxi, China \\ ${ }^{2}$ Shaanxi Research Center of Biopesticide Engineering \& Technology, Northwest A\&F University, Yangling, Shaanxi, China
}

\begin{abstract}
This study evaluated the potential of horseradish (Armoracia rusticana) oil (ARO) and eight isothiocyanates (propyl ITC [ProITC], isopropyl ITC [IsoproITC], n-butyl ITC [n-BuITC], 3-butenyl ITC [3-BeITC], phenyl ITC [PhITC], benzyl ITC [BzITC], 2-phenylethyl ITC [PhEITC], and allyl ITC [AITC]) as preservatives and antifungal agents for postharvest tomato disease control. Results showed that ARO and eight ITCs demonstrated antifungal activities against Botrytis cinerea, Alternaria alternata, Rhizopus stolonifer, and Geotrichum candidum, which can cause

and G. candidum, respectively. ARO, 2-PhEITC, BzITC, and AITC exhibited better efficacy as preservatives of mature green tomato than other ITCs on the basis of some parameters, such as low decay rate, slow reduction in weight loss, slight change in hardness, slow decrease in acidity, and total soluble solid content of treated tomatoes. GC-MS revealed that 2 -PhEITC $(77.78 \%)$ and AITC $(15.87 \%)$ were the major components of ARO. These results can be used as a basis to develop preservative products composed of ITCs.
\end{abstract} the decay of mature green tomato during storage. Allyl-ITC (AITC) had the lowest $\mathrm{EC}_{50}$ values of mycelia growth suppression, with 0.18 , $0.44,0.29$, and $0.43 \mu \mathrm{g} / \mathrm{ml}$ air for B. cinerea, A. alternata, $R$. stolonifer,

Keywords: Armoracia rusticana oil, isothiocyanates, antifungal, tomato, preservation

Tomatoes are cultivated in most provinces in China; however, tomatoes are a seasonal fruit and are susceptible to pathogenic fungal infection because of their high water content. Additionally, tomato fruit skin is thin and soft, and is easily damaged and decayed after harvest (Brady 1987). Fungal contamination and lipid peroxidation adversely affect the shelf life of tomato, resulting in significant economic losses during different stages after harvest. Botrytis cinerea, Alternaria alternata, Rhizopus stolonifer, and Geotrichum candidum are the main postharvest fungal pathogens of tomato fruit and are responsible for appreciable tomato losses (Kong et al. 2019; Prusky et al. 2001; Shi and Sun 2017; Talibi et al. 2012).

The use of synthetic preservatives is currently discouraged because of their potential undesirable biological effects, such as carcinogenic effects on animals and humans (Osman and Abdulrahman 2003). In recent years, numerous plant essential oils have been investigated to maintain the freshness of fruits and vegetables. Some plant essential oils, which are generally recognized as safe, demonstrate a wide range of antifungal activities (Prakash et al. 2012; Soliman and Badeaa 2002), increase the shelf life of fruits and vegetables (Akash et al. 2014), and induce host disease resistance. Some essential oilbased fungicides and shelf life-extending agents, such as ethylicin and eugenol, are registered in China (Ji et al. 2010; Lan et al. 2011). Isothiocyanates (ITCs) extracted from cruciferous plants exhibit better controlling efficacy against warehouse pests, pathogenic microorganisms, root-knot nematodes, and weeds (Wu et al. 2011). Wang et al. (2010) showed that Allyl ITC (AITC) was effective against blueberry decay during storage at $10^{\circ} \mathrm{C}$. Application of beta

${ }^{\dagger}$ Corresponding author: H. Wu; wgf20102010@nwsuaf.edu.cn

Funding: The authors are grateful to the Key R \& D Projects of Shaanxi Province (2020ZDLNY-07-01) and the Xi'an Science and Technology Bureau (20193063YF051NS051) for financial support.

The author(s) declare no conflict of interest.

Accepted for publication 6 April 2020.

(C) 2020 The American Phytopathological Society cyclodextrin entrapped allyl isothiocyanate $(200 \mu \mathrm{l} / \mathrm{liter})$ to packaged fresh-cut onions effectively decreased numbers of Listeria monocytogenes, which decreased at slower rates to undetectable levels on untreated cut onion (Piercey et al. 2012). Recent research has also showed that AITC microcapsule formulation can extend the shelf life of mature green tomato (Wu et al. 2015). However, AITC is just a member of the ITC family of Armoracia rusticana oil (ARO), and many ITCs can be obtained from horseradish oil. Further studies are required to investigate the potential of other ITCs as preservatives on mature green tomatoes. The objectives of this study were: (i) to ascertain the control effect of ARO and eight single ITC components for controlling postharvest fungal pathogens of tomato fruit; (ii) to determine the preservative effect of ARO and the eight ITCs on tomatoes; and (iii) to evaluate the potential value of ARO and single ITC components when applied as preservatives.

\section{Materials and Methods}

Chemicals and equipment. The pure isothiocyanate chemicals, propyl ITC (ProITC), isopropyl ITC, $n$-butyl ITC (n-BuITC), 3butenyl ITC (3-BeITC), phenyl ITC (PhITC), benzyl ITC (BzITC), 2-phenylethyl ITC (2-PhEITC), and AITC were obtained from Sigma-Aldrich Chemical Co. (St. Louis, MO). Dried roots of A. rusticana (moisture content $<10 \%$, storage under dry and shaded conditions), collected from Pengshi, Sichuan Province, China, were pulverized and passed through a 40 to 60 mesh uniform sieve before extraction. ARO was extracted from A. rusticana powder as previously described (Leoni et al. 1997; Wu et al. 2009).

Fruit materials. Tomatoes were harvested from commercial greenhouse-grown plants in Yangling, Shaanxi Province on 30 April 2015. These tomatoes were transported to laboratory and selected according to some characteristics, such as shape, size, color and luster, presence of stalk, absence of mechanical damage, and absence of pests on or in the tomatoes. The collected tomatoes were washed with biodegradable detergent, rinsed with tap water, and allowed to air dry at room temperature until further studies.

Fungal cultures. $B$. cinerea Pers. and A. alternata were provided by the Research \& Development Center of Biorational Pesticide, Northwest A \& F University (NWAFU). R. stolonifer and G. candidum were obtained from Wuhan Nature's Favor Bioengineering Co., 
Ltd. Fungi were cultured on potato dextrose agar (PDA). The PDA plates were incubated at $28^{\circ} \mathrm{C}$ on a $12 \mathrm{~h}$ dark $/ 12 \mathrm{~h}$ light cycle for 4 days.

Chemical characterization of ARO. ARO was analyzed through gas chromatography (Thermo Electron Corporation) equipped with the fused silica capillary column OV-17 $(30 \mathrm{~cm} \times 0.53 \mathrm{~mm} \times$ $0.5 \mu \mathrm{m})$. The oven was maintained at $60^{\circ} \mathrm{C}$ for $10 \mathrm{~min}$, and then increased to $200^{\circ} \mathrm{C}$ at a rate of $10^{\circ} \mathrm{C} / \mathrm{min}$. The injector and detector temperatures were both maintained at $250^{\circ} \mathrm{C}$. The injection volume was $0.1 \mu \mathrm{l} . \mathrm{H}_{2}$ and $\mathrm{N}_{2}$ were fed at a flow rate of $30 \mathrm{ml} / \mathrm{min}$, whereas air was fed at a flow rate of $300 \mathrm{ml} / \mathrm{min}$. Analysis was performed using MS (the ion trap temperature, manifold temperature, and transfer line temperature were set at $250^{\circ} \mathrm{C}$ ). The mass spectrum was automatically retrieved by the system mass spectrometer. The authentic standard (STD), mass spectrometry cleavage pattern, eight-peak index, peak-to-peak mass-to-charge ratio and relative abundance, National Institute of Standards and Technology (NIST/EPA/NIH), Spectral Database for Organic Compounds (SDBS) (https://sdbs.db.aist. go.jp/sdbs/cgi-bin/cre_list.cgi), and related literature were used to determine the individual components (Gilbert and Nursten 1972; Jiang et al. 2006).

Effects of ARO and of the eight ITCs on in vitro mycelial growth. Based on the results of the pretest, the eight ITCs and ARO were fumigated using serial concentrations in the range of 0.078 to $5 \mu \mathrm{g} / \mathrm{ml}$ air, which were taken or diluted in water (seven concentrations were used for mycelial growth trials: $0.078,0.156,0.313$, $0.625,1.25,2.5$, and $5 \mu \mathrm{g} / \mathrm{ml}$, vapor concentration of ITC in the closed desiccator). Before fumigation treatment, mycelia of fungal species B. cinerea, A. alternata, $R$. stolonifer, and G. candidum were cultured for $12 \mathrm{~h}$ by placing a block ( $4 \mathrm{~mm}$ diameter) obtained from an actively growing culture at the center of a 10-ml PDA Petri dish. These Petri dishes were placed in the upper level of a desiccator, which had been sterilized by $75 \%$ ethyl alcohol and UV (700 lux) 30 min before use. A water solution containing ITC, ARO, carbendazol, or solvent was transferred to the lower level of the desiccators. Subsequently, these desiccators were sealed with petroleum jelly. The fumigation treatments were performed for 3 to 5 days until the mycelia had reached the edge of the plate, and then the length of mycelial growth was measured using a centimeter ruler.

Carbendazol treatment (25\% carbendazim wettable powder purchased from Sichuan Blue Technology Co., Ltd.), diluted with dimethyl sulfoxide (DMSO) and water solution, was used as the positive control. The treatment method was as follows: $9 \mathrm{ml}$ PDA culture medium and $1 \mathrm{ml}$ prepared solution (including carbendazol) were added to Petri dishes to form a mixture containing carbendazol concentrations of $10,5,2.5,1.25,0.625,0.313$, and $0.156 \mu \mathrm{g} / \mathrm{ml}$, and the final concentration of DMSO was lower than $1 \%(\mathrm{v} / \mathrm{v})$. The solvent and water mixture was set as the blank control. Fungi blocks ( $4 \mathrm{~mm}$ ) of these four fungal species were inoculated on Petri dishes. The mycelial growth lengths were measured according to the above description.

Each treatment was replicated four times for each fungal species (Fig. 1). The experiment was repeated three times and representative data from one of the experiments was shown. The mycelial growth inhibition ratio of each treatment (desiccator) against each fungal species was assessed separately by equation 1 :

Mycelial growth inhibition ratio $(\%)=$

$\underline{\text { growth diameter of control }(\mathrm{mm}) \text { - growth diameter of treatment }(\mathrm{mm})}$ growth diameter of control $(\mathrm{mm})-4(\mathrm{~mm})$

Evaluation of the preservation activity of eight ITCs and ARO on tomatoes. ITCs and ARO $(1,000 \mu \mathrm{g} / \mathrm{ml})$ were prepared by using $0.05 \%$ Tween aqueous solution. A treatment of $0.05 \%$ Tween aqueous solution was used as the blank control and $25 \%$ carbendazim wettable powder was used as positive control. Various chemicals (eight ITCs), along with the positive control, were diluted to $500 \mu \mathrm{g} / \mathrm{ml}$ and prepared tomatoes were dipped for 3 to $5 \mathrm{~s}$ in these diluted solutions. After drying at 25 to $28^{\circ} \mathrm{C}$ for $4 \mathrm{~h}$, the treatment tomatoes were placed into a plastic bag $(($ wide + sides $) \times$ height $\times$ thickness $\left.=(300+120) \mathrm{mm} \times 500 \mathrm{~mm} \times 0.05 \mathrm{~mm}, \rho=1.38 \mathrm{~g} / \mathrm{cm}^{3}\right)$ and sealed as a repeat, and each treatment (12 tomatoes) was repeated three times. The four marked tomatoes in each treatment were used to detect weight loss at an interval of 5 days. All treatment tomatoes were stored at room temperature. The experiment was repeated three times and representative data from one of the experiments was shown.

The percent rotten rate was calculated by Equation 2, weight loss was calculated by Equation 3, and the hardness was determined at the end of each storage period using Equation 4. The total soluble solid (TSS) content was measured by an automatic refract meter/sugar meter RA-600 (Kyoto Electronics Manufacturing Co., Ltd). Titratable acid ( $\mathrm{pH}$ titration) of tomato was determined according to the following procedures: The fresh fruit $(5 \mathrm{~g})$ were ground in the mortar until a homogenized fraction was obtained. Then, the homogenate was transferred into distilled water to $100 \mathrm{ml}$ and warmed in an $80^{\circ} \mathrm{C}$ water bath for $30 \mathrm{~min}$. After cooling, the juice was filtered through three layers of cheesecloth. The TA was determined by titrating $20 \mathrm{ml}$ of the filtrate with $0.1 \mathrm{M}$ sodium hydroxide standard solution with phenolphthalein indicator.

$$
\begin{aligned}
\text { Percent rotten }(\%) & =\frac{\text { Number of rotten fruits }}{\text { Total fruits }} \times 100 \\
\text { Weight loss }(\%) & =\frac{\text { Initial weight-Final weight }}{\text { Initial weight }} \times 100 \\
\text { Hardness }\left(\mathrm{kg} / \mathrm{cm}^{2}\right) & =\frac{x(\mathrm{~kg})}{\pi r^{2}} \\
\mathrm{r} & =0.5 \mathrm{~cm}
\end{aligned}
$$

Statistical analysis. The values of $\mathrm{EC}_{50}$ and $95 \%$ confidence limit of eight ITCs and ARO against in vitro mycelial growth were determined by SPSS 25.0. Data were tested for normality before performing mean separation, and separation of means was performed using the least significant difference test, at $P<0.05$. Mean comparisons and standard deviations were calculated according to Duncan's multiple range test.

\section{Results}

Effects of eight ITCs and ARO on in vitro mycelial growth. The ARO and the eight ITCs showed fungicidal activity against mycelial growth of B. cinerea, A. alternata, R. stolonifer, and G. candidum. Compared with the other ITCs and carbendazol, AITC had the lowest $\mathrm{EC}_{50}$ values of $0.18,0.44,0.29$, and $0.43 \mu \mathrm{g} / \mathrm{ml}$ air against mycelial growth of B. cinerea, A. alternata, R. stolonifer, and G. candidum, respectively (Table 1). Besides that, ARO was also excellent in controlling the mycelial growth of the four fungal species compared with carbendazol treatment.

GC/MS analysis. The major components of this oil were found to be allyl thioayanate, AITC, n-BuITC, 3-butenyl ITC, n-amyl ITC, 5-hexenyl ITC, benzyl cyanide, BzITC, 2-phenylethyl ITC, and an unknown compound. Relative quantitative analysis of the ARO (chromatographic peak area normalization method) indicated that

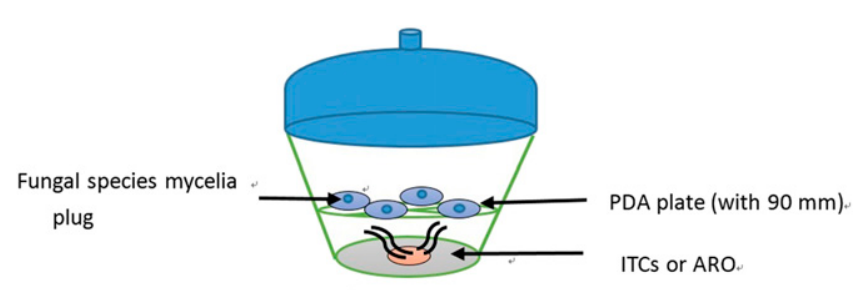

Fig. 1. Schematic diagram of Armoracia rusticana oil (ARO) or isothiocyanate (ITC) fumigation technique used in the study. 
the oil mainly contained 2-phenylethyl ITC (77.78\%), AITC (15.87\%), and allyl thioayanate $(2.18 \%)$, respectively, and the results are shown in Figure 2.

Effect of ARO and eight ITCs on tomato percent rotten. Table 2 shows the percent rotten of the ARO and ITC-treated tomatoes, positive control, and blank control. The results show that some ITCs such as ProITC, AITC, ARO, and BzITC had positive effects on the quality of tomatoes. Compared with that of the blank control and the carbendazol-treated tomatoes, low percent rotten was observed in the 2-PhEITC-, BzITC-, and ARO-treated tomatoes.

Table 1. Effects of eight isothiocyanates (ITCs) and Armoracia rusticana oil (ARO) on mycelia growth in vitro ${ }^{z}$

\begin{tabular}{|c|c|c|c|c|}
\hline \multirow[b]{2}{*}{ Treatment } & \multicolumn{4}{|c|}{$\mathrm{EC}_{50}(\mu \mathrm{g} / \mathrm{ml})(95 \%$ confidence limits $)$} \\
\hline & B. cinerea & A. alternata & R. stolonifer & G. candidum \\
\hline ProITC & $0.60(0.51-0.66) b$ & $0.89(0.72-1.01) b$ & $3.89(3.57-4.23) \mathrm{e}$ & $4.87(4.44-5.10) \mathrm{f}$ \\
\hline IsoProITC & $1.19(0.92-1.26) \mathrm{cd}$ & $1.45(1.32-1.53) \mathrm{d}$ & $5.32(5.27-5.42) \mathrm{f}$ & $3.65(3.32-3.76) \mathrm{e}$ \\
\hline n-BuITC & $0.86(0.78-0.93) \mathrm{c}$ & $0.77(0.63-0.82) b$ & $2.57(2.42-2.66) \mathrm{d}$ & $2.33(2.24-2.92) \mathrm{d}$ \\
\hline 3-BeITC & $0.99(0.93-1.13) \mathrm{cd}$ & $1.11(0.99-1.52) \mathrm{c}$ & $3.37(3.44-3.56) \mathrm{e}$ & $3.35(3.24-3.92) \mathrm{e}$ \\
\hline AITC & $0.18(0.12-0.23) \mathrm{a}$ & $0.44(0.35-0.58) \mathrm{a}$ & $0.29(0.21-0.44) \mathrm{a}$ & $0.43(0.31-0.49)$ a \\
\hline PhITC & $1.53(1.49-1.59) \mathrm{d}$ & $1.23(1.09-1.44) \mathrm{c}$ & $0.78(0.57-0.89) b$ & $1.54(1.27-1.67) \mathrm{c}$ \\
\hline BzITC & $1.09(0.92-1.16) \mathrm{cd}$ & $1.17(1.06-1.26) \mathrm{c}$ & $0.43(0.38-0.55) \mathrm{a}$ & $0.67(0.52-0.73) b$ \\
\hline 2-PhEITC & $2.21(2.01-2.33) \mathrm{e}$ & $1.67(1.53-1.83) \mathrm{d}$ & $1.57(1.42-1.73) \mathrm{c}$ & $2.55(2.33-2.72) \mathrm{d}$ \\
\hline ARO & $0.23(0.19-0.28) \mathrm{a}$ & $0.34(0.24-0.45) \mathrm{a}$ & $0.33(0.31-0.47) \mathrm{a}$ & $0.32(0.22-0.42)$ a \\
\hline Carbendazol & $1.56(1.32-1.87) \mathrm{d}$ & $1.25(1.01-1.47) \mathrm{c}$ & $1.42(1.26-1.63) \mathrm{c}$ & $1.12(1.06-1.32) \mathrm{c}$ \\
\hline
\end{tabular}

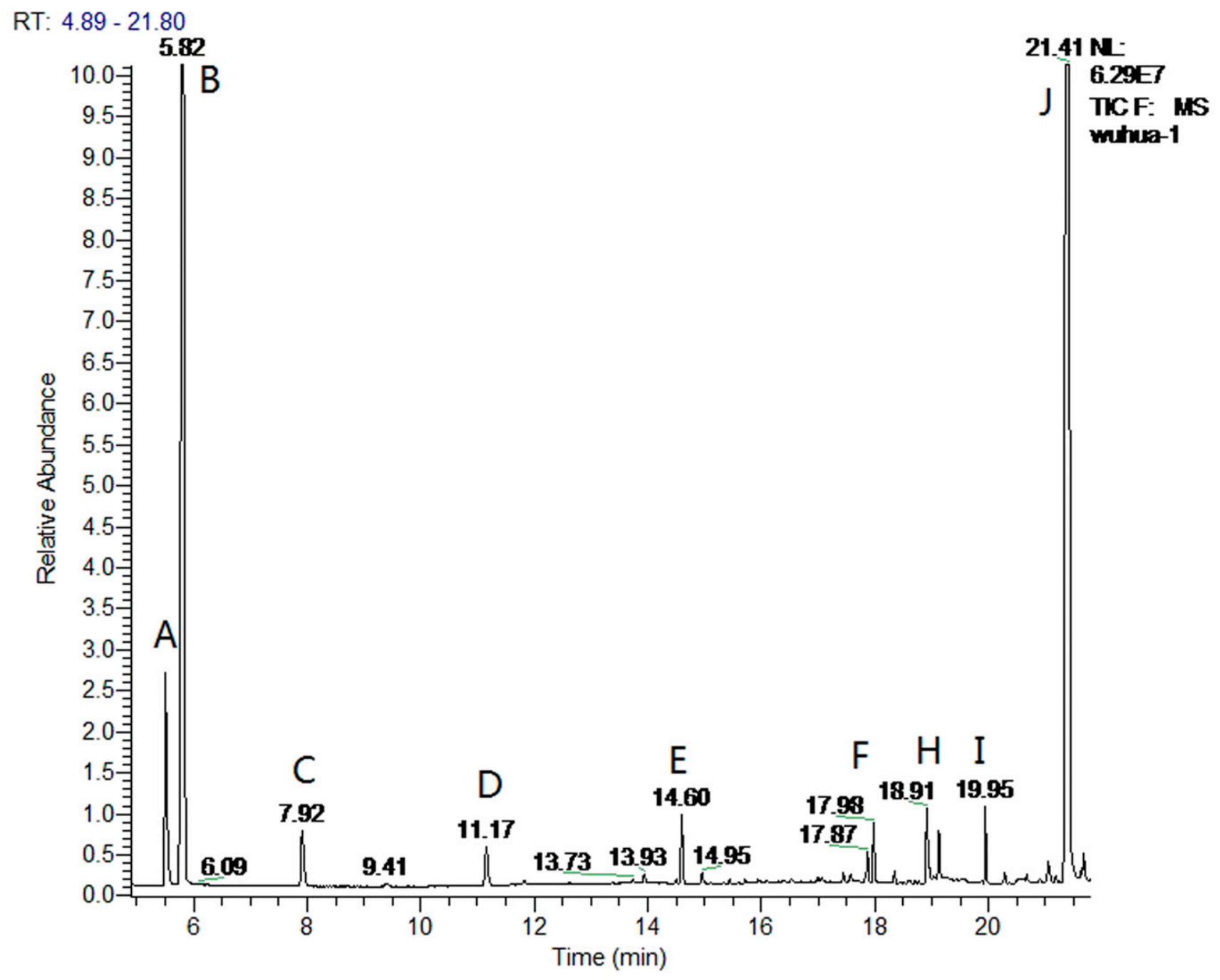

Fig. 2. GC/MS of Armoracia rusticana oil (ARO): Allyl thioayanate (A, 2.18\%), AITC (B, 15.87\%), n-BulTC (C, $0.60 \%)$, 3-butenyl ITC (D, $0.43 \%)$, amyl ITC (E, $0.67 \%)$, hexenyl ITC $(\mathrm{F}, 0.56 \%)$, benzyl cyanide $(\mathrm{G}, 0.71 \%)$, unknown $(\mathrm{H}, 0.50 \%)$, BzITC $(\mathrm{I}, 0.71 \%)$, and 2-phenylethyl ITC $(\mathrm{J}, 77.78 \%)$. 
The 30-day total percent rotten of 2-PhEITC-, BzITC-, and AROtreated tomatoes was only $8.3 \%$, while the 30 -day total percent rotten of the positive control and blank control treated tomatoes were $19.4 \%$ and $13.9 \%$, respectively. Moreover, there were significant differences $(P=0.05)$ in 30-day total percent rotten between single 2PhEITC-, BzITC-, and ARO-treated and positive control and blank control treated tomatoes (Table 2).

Effect of eight ITCs and ARO on weight loss and hardness of tomatoes. Figures 3 and 4 show the rate of weight loss and change in hardness, respectively, of the tomatoes treated with ARO and with the eight ITCs. The weight loss for different tomato treatments increased with storage time (Fig. 3). The $n$-BuITC and PhITC treatment groups, along with the blank control group, showed the fastest increase in weight loss, and the total weight loss of the tomatoes after 30 days was $3.13,3.34$, and $3.26 \%$, respectively. No significant difference $(P=0.05)$ in weight loss was observed among them after 30 days. In contrast, ProITC, AITC, BzITC, 2-PhEITC, and
ARO treatment groups showed slow increase in weight loss rate $(P=0.05)$, and the accumulated weight loss of the tomatoes after 30 days was $2.39,2.34,2.52,2.65$, and $2.42 \%$, respectively. The weight loss in the positive control $(3.12 \%)$ was similar to that of the blank group. On the other hand, the hardness of the tomatoes decreased gradually with storage time (Fig. 4). The fruit hardness in ARO, 2-PhEITC, BzITC, and AITC treatment groups decreased more slowly than the control and the carbendazol treatment groups. After 10 days, no significant difference $(P=0.05)$ in fruit hardness (average value about $4.5 \mathrm{~kg} / \mathrm{cm}^{2}$ ) was observed among the groups treated with various ITCs, the positive control, and the blank control. After 30 days, however, the hardness of tomatoes of ARO, 2-PhEITC, BzITC, and AITC treatment were $2.43,2.39,2.38$, and $2.35 \mathrm{~kg} / \mathrm{cm}^{2}$, which were significantly higher $(P=0.05)$ than the other treatment groups $\left(1.80\right.$ to $\left.2.0 \mathrm{~kg} / \mathrm{cm}^{2}\right)$.

Effect of eight ITCs and ARO on TSS and acidity of tomatoes. Figures 5 and 6 show the TSS and acidity, respectively, of the

Table 2. Effect of treatment of eight isothiocyanates (ITCs) and Armoracia rusticana oil (ARO) on tomato rotting rate during storage ${ }^{\mathrm{z}}$

\begin{tabular}{|c|c|c|c|c|c|}
\hline \multirow[b]{2}{*}{ Treatment } & \multicolumn{4}{|c|}{ Total number of decayed tomatoes } & \multirow[b]{2}{*}{ Total rotting rate after 30 days \pm S.E. $(\%)$} \\
\hline & 15 days & 20 days & 25 days & 30 days & \\
\hline ProITC & 0 & 1 & 2 & 5 & $13.9 \pm 2.78 \mathrm{bc}$ \\
\hline IsoProITC & 0 & 1 & 3 & 5 & $13.9 \pm 2.78 \mathrm{bc}$ \\
\hline n-BuITC & 0 & 0 & 3 & 6 & $16.7 \pm 0.00 \mathrm{ab}$ \\
\hline 3-BeITC & 0 & 0 & 2 & 5 & $13.9 \pm 2.78 \mathrm{bc}$ \\
\hline AITC & 0 & 1 & 1 & 4 & $11.1 \pm 2.78 \mathrm{bc}$ \\
\hline PhITC & 0 & 2 & 2 & 7 & $19.4 \pm 2.78 \mathrm{a}$ \\
\hline BzITC & 0 & 0 & 1 & 3 & $8.3 \pm 0.00 \mathrm{~cd}$ \\
\hline 2-PhEITC & 0 & 0 & 2 & 3 & $8.3 \pm 0.00 \mathrm{~cd}$ \\
\hline ARO & 0 & 0 & 1 & 3 & $8.3 \pm 0.00 \mathrm{~cd}$ \\
\hline Blank control & 0 & 2 & 3 & 5 & $13.9 \pm 2.78 \mathrm{ab}$ \\
\hline Positive control & 0 & 2 & 3 & 7 & $19.4 \pm 2.78 \mathrm{a}$ \\
\hline
\end{tabular}

${ }^{\mathrm{z}}$ The concentration of each treatment was set at $500 \mu \mathrm{g} / \mathrm{ml}$. Data with different letters in the same column indicate significant difference at the $5 \%$ level.

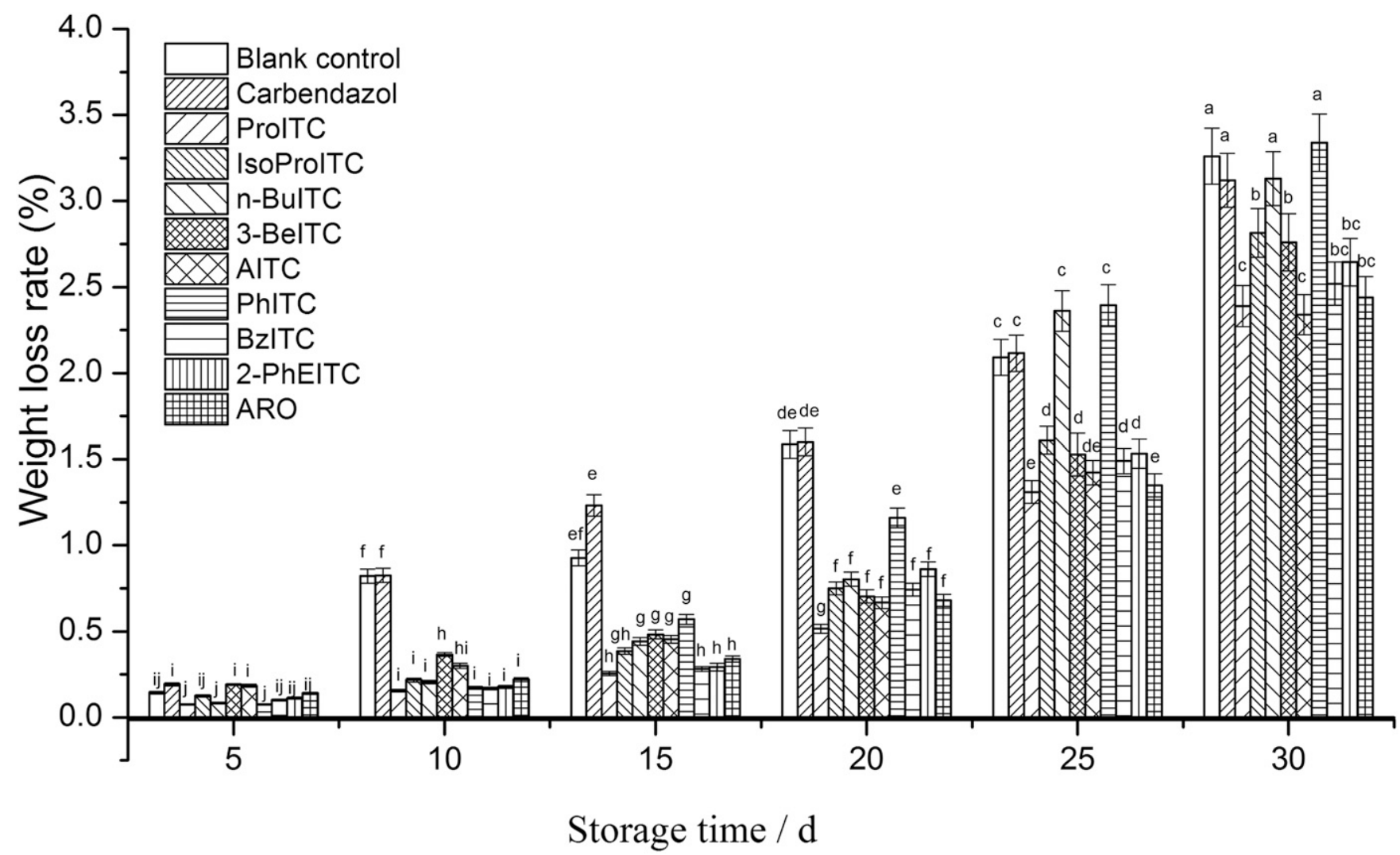

Fig. 3. Weight loss rate change during tomato storage. Data bars are mean $\pm \mathrm{SE}, n=3$. 


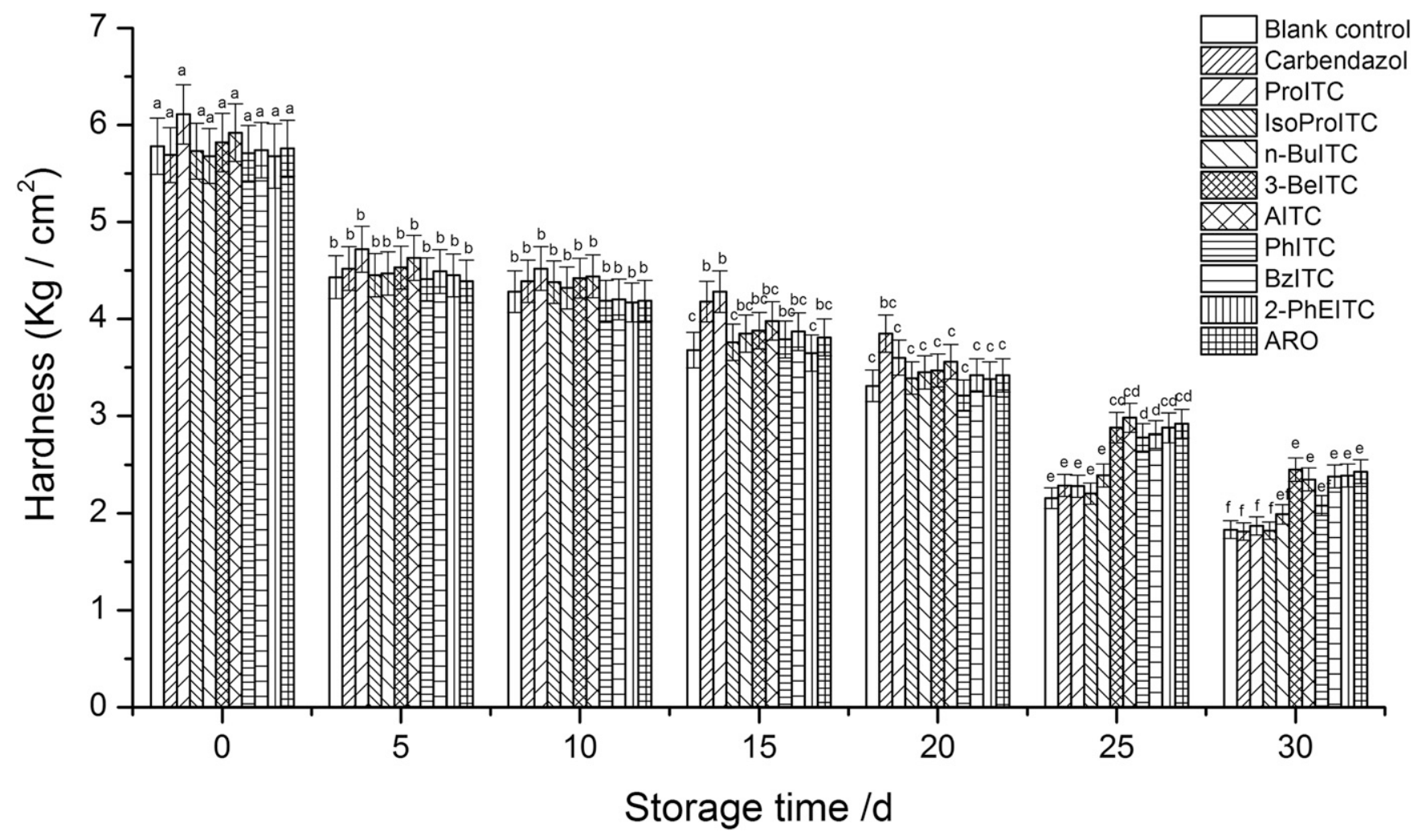

Fig. 4. Hardness change during tomato storage. Data bars are mean $\pm \mathrm{SE}, n=3$.

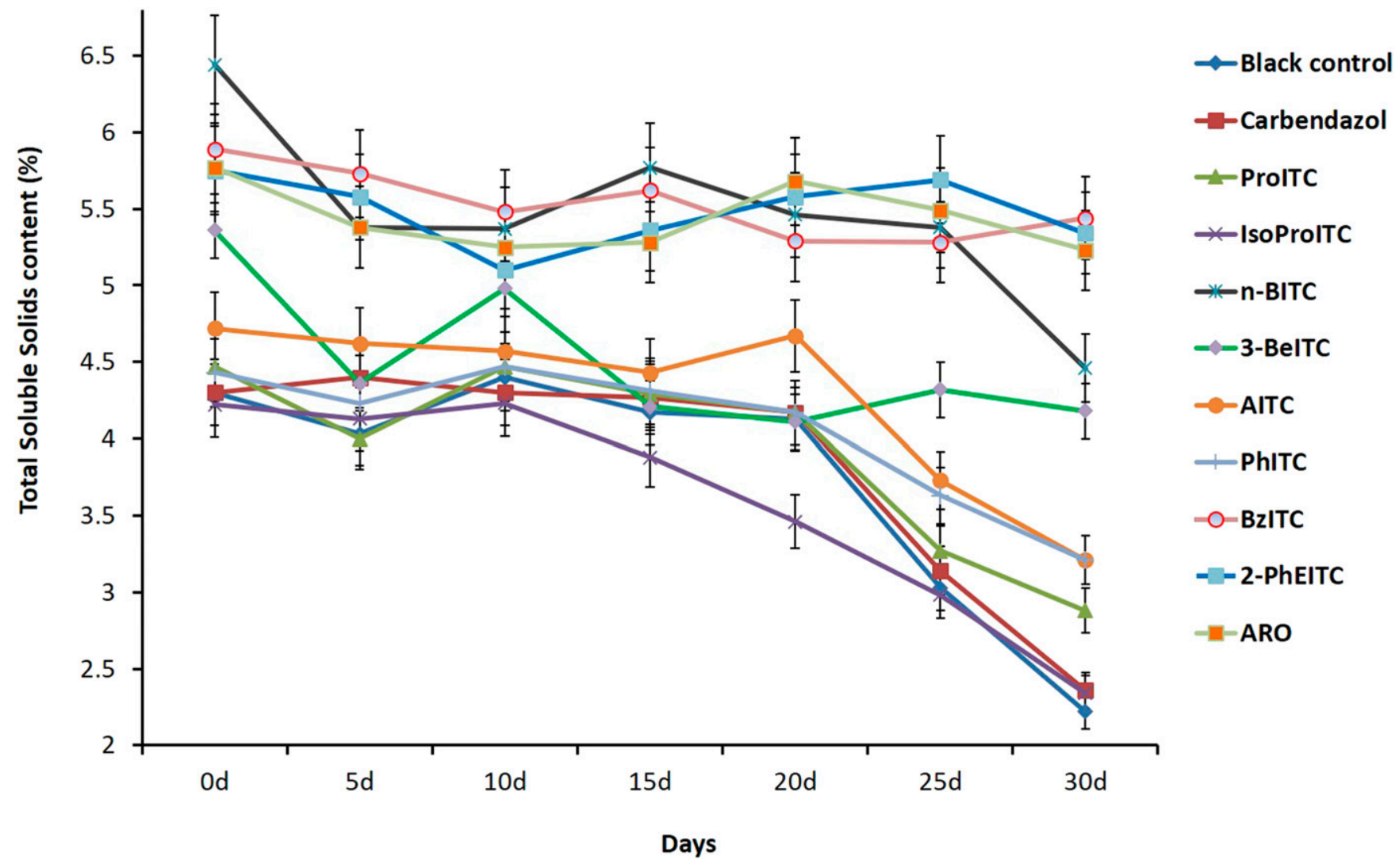

Fig. 5. Change in the content of total soluble solids in tomato during storage after treatment with eight isothiocyanates (ITCs) and Armoracia rusticana oil (ARO). Data bars are mean \pm SE, $n=3$. 
tomatoes treated with ARO and with the eight ITCs. The TSS of the control group and those treated with ARO and ITCs showed a downward trend after the initial upward trend (Fig. 5). The decreases in TSS were faster in the blank control and the positive control group than in the treatment groups during the 30 days, which dropped from 4.3 to $2.22 \%$, and from 4.3 to $2.36 \%$, respectively. In contrast, the TSS of the ARO, 2-PhEITC, and BzITC treatment groups decreased slower than in the control groups. During the 30 days, the TSS of tomatoes of the ARO, 2-PhEITC, and BzITC treatment groups decreased from 5.77 to $5.23 \%$, from 5.75 to $3.4 \%$, and from 5.89 to $5.44 \%$, respectively. After the 30 days, moreover, the TSS of tomatoes of ARO, 2-PhEITC, BzITC, and AITC treatment were significantly higher $(P=0.05)$ than the other treatment groups $(2.22$ to $4.46 \%$ ).

During the 30 days, the change in acidity of tomatoes of the positive control and the blank control treatment increased at first and then decreased, while the change in acidity of tomatoes of ARO, 2-PhEITC, and BzITC treatment initially dropped, followed by an increase, before finally showing a descending trend. The change in acidity of the other ITC-treated tomatoes fluctuated. After 30 days, moreover, the change in acidity in ARO, 2-PhEITC, BzITC, and ProITC treatment groups were significantly slower $(P=0.05)$ than in other groups. Some ITCs also obviously inhibited changes in tomato acidity (Fig. 6).

\section{Discussion}

Essential oils derived from aromatic and medicinal plants are potentially useful as antimicrobial agents, and their application as medicines has long been recognized (Laranjo et al. 2019). Currently, food researchers are attracted to botanical products because of their ability to reduce the amount of synthetic ingredients in foods. Moreover, the studies on botanical products as shelf life-extending agents, which are generally recognized as safe products, may lead to the exploitation of essential oils for this purpose (Smid and Gorris 1999).
Biologically friendly plant-derived essential oils are apparently popular. Thus, the use of essential oils as antifungal or/and shelf life-extending agents is receiving increased attention worldwide. Akash et al. (2014) reported the antifungal property of cumin seed essential oil and its efficacy as fumigant in food systems. Plant essential oils extracted from Agastache mexicana ssp. xolocotziana and Porophyllum linaria could exert inhibitory effects against 11 different fungal strains isolated from stored wheat grains (Juárez et al. 2015). Numerous studies also reported essential oils as a preservative for fresh fruits and food systems. Prakash et al. (2012) evaluated some essential oils used as food preservatives in terms of their antifungal, antiaflatoxin, and antioxidant activities, as well as their in vivo efficacy in the food system. Many studies have also reported the potential use of plant-derived ITCs as biofumigant fungicides and their application in storage environments (Azaiez et al. 2013; Mari et al. 2002, 2008; Troncoso et al. 2005); however, very few studies have focused on the potential of ITCs as preservatives.

The AROs from different horseradish cultivars have different chemical profiles, as well as distinct activities (Mari et al. 1996, 2008). The main variations of ARO are the components and proportion changes of ITCs (Gilbert and Nursten 1972; Jiang et al. 2006). Some of them are easy to volatilize, such as short chain saturated alkyl ITC and unsaturated hydrocarbon ITC and show good fumigation against pests, while aryl ITC and indolyl ITC have demonstrated contact toxicology against pests. According to Lin et al. (2001), the major components of ARO extracted from horseradish cultivation species as analyzed by GC/MS were AITC (31.83\%) and 4-pentenyl isothiocyanate (26.24\%); however, 2-PhEITC (77.78\%) and AITC $(15.87 \%)$ were detected as major components in the current study.

Tomatoes are naturally covered by a continuous wax layer that provides high resistance to water movement across the cuticle. The fumigation treatment with single ITC chemicals can partly delay weight loss and improve the commodity rate of tomato; these effects may be related to the ability of ITC to prevent increase in local

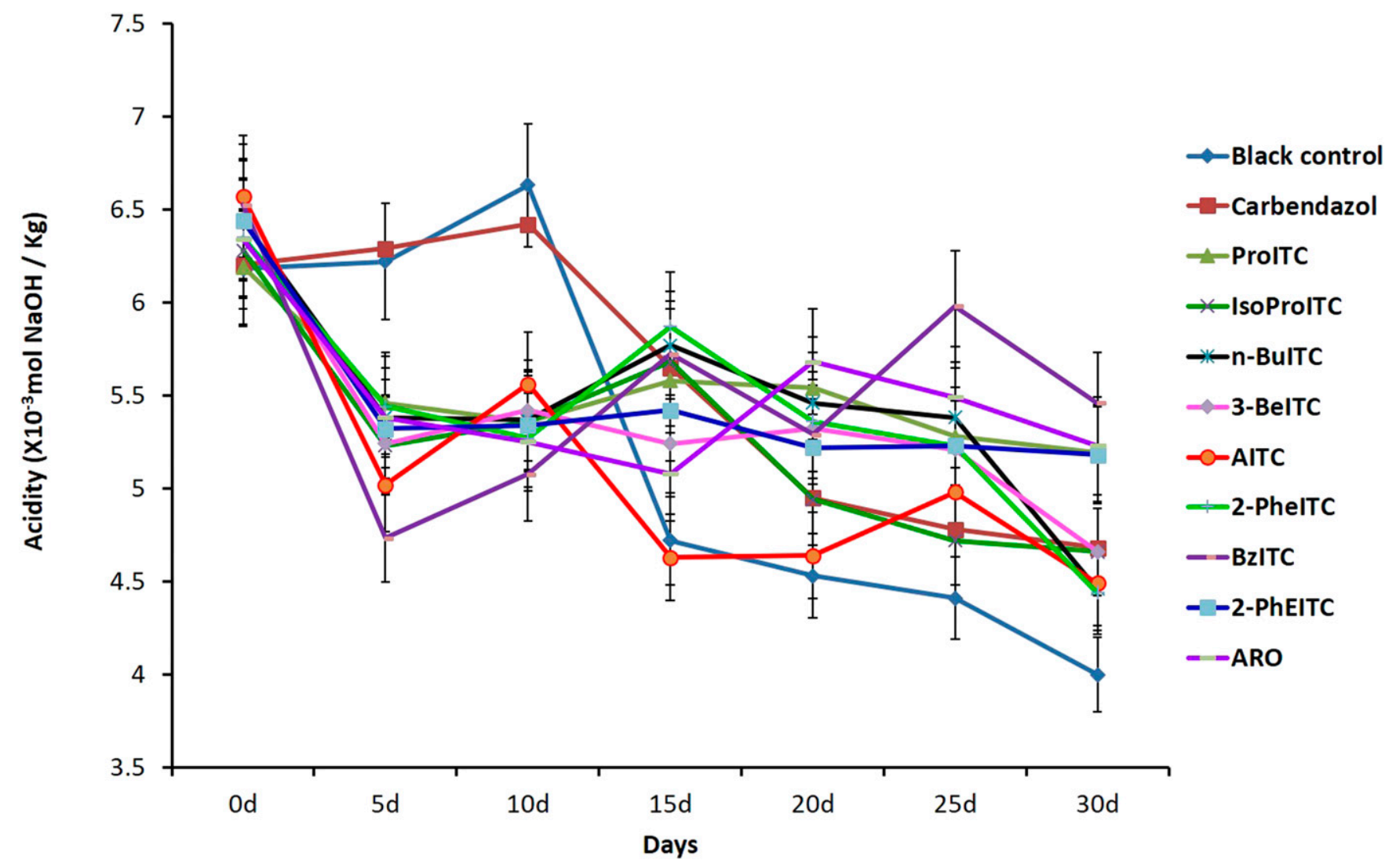

Fig. 6. Acidity change after treatment of eight isothiocyanates (ITCs) and Armoracia rusticana oil (ARO) in tomato during storage. Data bars are mean \pm SE, $n=3$. 
saturated vapor pressure of tomato, delay green tomato fruit postripening, and reduce the respiratory intensity of tomato fruit. Other studies have reported that plant essential oil chemicals, essential oil nano-preparation (microcapsule preparation), or essential oil coating treatment can effectively control the loss of water in fruits and vegetables (Fagundes et al. 2014; Wu et al. 2015). The sensory and internal qualities of tomato are considered the most important parameters affecting consumers' acceptance (Grierson and Kader 1986). Fumigation treatment with different ITCs significantly affected the hardness, TA, and SSC of tomatoes in the current study, especially for the ARO and its main constituents (AITC and 2PhEITC). These compounds are possibly related to the conversion of starch into sugar in mature green tomatoes, which may delay the maturation of the tomato and consequently extend its shelf life. Other research results also demonstrate that some formulations or coatings based on essential oils can significantly affect the parameters of fruits and vegetables (Das et al. 2013; Fagundes et al. 2014). We also believe that the main reasons behind the differences observed include differences in treatment methods (fumigation and soaking), which could block the different ripening pathways of the fruits.

This study, on one hand, revealed the fumigation activity of ITCs and ARO against the pathogenic fungi in tomato, and on the other hand, evaluated ITC and ARO as preservatives and particularly assessed the weight loss and percent rotten, as well as the changes in acidity and TSS during tomato storage. The preservative activity of AITC are reported (Wu et al. 2015), but the relationship between the ARO and single ITC components deserves an attention for application as preservatives. ARO includes the unsaturated hydrocarbon ITCs, saturated hydrocarbon ITCs, and the aromatic ITCs (Jiang et al. 2006). AITC and EITC exhibit synergistic interaction, resulting in strong antifungal activity (Wu et al. 2011). Although BzITC is not contained in the ARO, it also demonstrates a better preservation activity on tomato storage in this research. As many plant essential oils possess compounds with similar structures, their combinations may exhibit additive rather than synergistic effects (Dorman and Deans 2000). Whether there is a synergistic interaction between AITC and 2-PEITC, or between BzITC and AITC, needs to be studied.

Variations in the amount of active oxygen and free radicals, as well as the enzymatic defense system, are associated with decay of fruits and vegetables (Fan et al. 2019). Active oxygen free radicals exert a strong oxidation effect, causing cell membrane peroxidation that disrupts biological membranes and increases membrane permeability and relative conductivity, resulting in unrepeatable metabolism, defunctionalization, and cell death (Chen 1992; Zhou et al. 2002). The plant enzymatic defense system mainly includes superoxide dismutase, peroxidase, catalase, APX (ascorbic acid), GST-PX (glutathion peroxidase), and GST-R (glutathione reductase) (Vardhini and Anjum 2015). As fruits and vegetables mature and decay, the enzymatic activity of the defense system is reduced and the balanced concentration of free radicals within the matrix is disrupted, leading to the accumulation of free radicals and rapid decay of fruit and vegetables (Rabinowitch and Sklan 1980). Certain concentrations of ITCs may activate the relevant enzymatic defense system of an organism (Wu et al. 2014) and can maintain the free radicals at low amounts, thereby retaining the freshness of the tomato. However, the mechanism of the preservative property of ITCs still requires further research.

This study simultaneously investigated the antifungal and preservative properties of ITCs and ARO, which could control the partial physiological and biochemical indices of tomato for pickling. Further studies should identify the ITCs that exhibit preservative activity and induce defense responses in fruits and vegetables. ITCs should be considered because these substances can be used to enhance the nutrient contents of fruits and combine fruit protection products for comprehensive development and utilization.

In conclusion, the antifungal activities and the preservative properties of ITCs were confirmed. AITC showed the lowest EC $_{50}$ values in mycelial growth of the investigated fungal species. Compared with the other ITCs, BzITC and ProITC exhibited better preservation activities as seen mainly in the relatively low decay rate, slowly reduced weight loss, and slow changes in hardness, acidity, and TSS. ARO showed good antifungal activities and preservative property, which is worthy of in-depth study. Antifungal activities and preservative activities of ITCs in combinations are worthy of further study.

\section{Literature Cited}

Akash, K., Bhanu, P., Prashant, K. M., and Dubey, N. K. 2014. Antifungal and antiaflatoxigenic properties of Cuminum cyminum (L.) seed essential oil and its efficacy as a preservative in stored commodities. Int. J. Food Microbiol. 168-169:1-7.

Azaiez, I., Meca, G., Manyes, L., Luciano, F. B., and Fernández-Franzón, M. 2013. Study of the chemical reduction of the fumonisins toxicity using allyl, benzyl and phenyl isothiocyanate in model solution and in food products. Toxicon 63:137-146.

Brady, C. J. 1987. Fruit ripening. Annu. Rev. Plant Physiol. 38:155-178.

Chen, S. Y. 1992. Low temperature stress and peroxidation of membrane lipid in sugarcane. J. Fujian Agric. For. Univ. 1:24-28 (Natural Science Edition).

Das, D. K., Dutta, H., and Mahanta, C. L. 2013. Development of a rice starchbased coating with antioxidant and microbe-barrier properties and study of its effect on tomatoes stored at room temperature. LWT-Food. Sci. Technol. 50:272-278.

Dorman, H. J. D., and Deans, S. G. 2000. Antimicrobial agents from plants: antibacterial activity of plant volatile oils. J. Appl. Microbiol. 88:308-316

Fagundes, C., Palou, L., Monteiro, A. R., and Pérez-Gago, M. B. 2014. Effect of antifungal hydroxypropyl methylcellulose-beeswax edible coatings on gray mold development and quality attributes of cold-stored cherry tomato fruit. Postharvest Biol. Technol. 92:1-8.

Fan, X. J., Zhang, B., Yan, H., Feng, J. T., Ma, Z. Q., and Zhang, X. 2019. Effect of lotus leaf extract incorporated composite coating on the postharvest quality of fresh goji (Lycium barbarum L.) fruit. Postharvest Biol. Technol. 148:132-140.

Gilbert, J., and Nursten, H. E. 1972. Volatile constituents of horseradish roots. J Sci. Food Agric. 23:527-539.

Grierson, D., and Kader, A. A. 1986. Fruit ripening and quality. Pages 241-280 in: The Tomato Crop-A Scientific Basis for Improvement. J. G. Atherton and J. Rudich, eds. Chapman \& Hall, London.

Ji, M. X., Wu, X., Shu, Z. L., Fei, Z. H., Chen, Y., and Miao, K. 2010. Test of new botanical fungicide-ethylicin•eugenol $20 \%$ WP for controlling strawberry gray mold caused by Botrytis cinerea. Agrochemicals. 49:458-459.

Jiang, Z. T., Li, R., and Yu, J. C. 2006. Pungent components from thioglucosides in Armoracia rusticana grown in China, obtained by enzymatic hydrolysis. Food Technol. Biotechnol. 1:41-45.

Juárez, Z. N., Hernandez, L. R., Bach, H., Sanchez-Arreola, E., and Bach, H. 2015 Antifungal activity of essential oils extracted from Agastache mexicana ssp. xolocotziana and Porophyllum linaria against post-harvest pathogens. Ind. Crops Prod. 74:178-182.

Kong, J., Zhang, Y., Ju, J., Xie, Y. F., Guo, Y. H., Cheng, Y. L., Qian, H., Quek, S. Y., and Yao, W. R. 2019. Antifungal effects of thymol and salicylic acid on cell membrane and mitochondria of Rhizopus stolonifer and their application in postharvest preservation of tomatoes. Food Chem. 285:380-388.

Lan, C. Z., Li, B. J., Zhao, J., Chen, Q. H., and Weng, Q. Y. 2011. Control efficiency of botanic fungicide on hot pepper Phytophthora blight. J. ChangJiang Vegetables 24:55-56.

Laranjo, M., Fernández-León, A. M., Agulheiro-Santos, A. C., Potes, M. E., and Elias, M. 2019. Essential oils of aromatic and medicinal plants play a role in food safety. J. Food Process. Preserv. doi.org/10.1111/jfpp.14278

Leoni, O., Iori, R., Palmieri, S., Esposito, E., Menegatti, E., Cortesi, R., and Nastruzzi, C. 1997. Myrosinase-generated Isothiocyanates from glucosinolates: isolation, characterization and in vitro antiproliferative studies. Bioorg. Med. Chem. 5:1799-1806.

Lin, X. H., Li, R., and Jiang, Z. T. 2001. Studies on the chemical component of horseradish essential oils. Food Sci. 22:373-375.

Mari, M., Iori, R., Leoni, O., and Marchi, A. 1996. Bioassays of glucosinolate-derived isothyocyanates against postharvest pear pathogens. Plant Pathol. 45:753-760.

Mari, M., Leoni, O., Bernardi, R., Neri, F., and Palmieri, S. 2008. Control of brown rot on stonefruit by synthetic and glucosinolate-derived isothiocyanates. Postharvest Biol. Technol. 47:61-67.

Mari, M., Leoni, O., Iori, R., and Cembali, T. 2002. Antifungal vapour-phase activity of allyl-isothiocyanate against Penicillium expansum on pears. Plant Pathol. 51:231-236.

Osman, K. A., and Abdulrahman, H. T. 2003. Risk assessment of pesticide to human and the environment. Saudi J. Biol. Sci. 10:81-106.

Piercey, M. J., Mazzanti, G., Budge, S. M., Delaquis, P. J., Paulson, A. T., and Truelstrup, H. L. 2012. Antimicrobial activity of cyclodextrin entrapped allyl isothiocyanate in a model system and packaged fresh-cut onions. Food Microbiol. 30:213-218.

Prakash, B., Singh, P., Kedia, A., and Dubey, N. K. 2012. Assessment of some essential oils as food preservatives based on antifungal, antiaflatoxin, antioxidant activities and in vivo efficacy in food system. Food Res. Int. 49: 201-208. 
Prusky, D., Eshel, D., Kobiler, I., Yakoby, N., Beno-Moualem, D., Ackerman, M., Zuthji, Y., and Arie, R. B. 2001. Postharvest chlorine treatments for the control of the persimmon black spot disease caused by Alternaria alternata. Postharvest Biol. Technol. 22:271-277.

Rabinowitch, H. D., and Sklan, D. 1980. Superoxide dismutase: A possible protective agent against sunscald in tomatoes. Planta 148:162-167.

Shi, J. F., and Sun, C. Q. 2017. Isolation, identification, and biocontrol of antagonistic bacterium against Botrytis cinerea after tomato harvest. Braz. J. Microbiol. 48:706-714.

Smid, E. J., and Gorris, L. G. M. 1999. Natural antimicrobials for food preservation. Pages 285-308 in: Handbook of Food Preservation. M. S. Rahman, ed. Marcel Dekker, New York.

Soliman, K. M., and Badeaa, R. I. 2002. Effect of oil extracted from some medicinal plants on different mycotoxigenic fungi. Food Chem. Toxicol. 40:1669-1675.

Talibi, I., Askarne, L., Boubaker, H., Boudyach, E. H., Msanda, F., Saadi, B., and Aoumar, A. A. B. 2012. Antifungal activity of some Moroccan plants against Geotrichum candidum, the causal agent of postharvest citrus sour rot. Crop Prot. 35:41-46.

Troncoso, R., Espinoza, C., Sánchez-Estrada, A., Tiznado, M. E., and Hugo, S. G. 2005. Analysis of the isothiocyanates present in cabbage leaves extract and their potential application to control Alternaria rot in bell peppers. Food Res. Int. 38: 701-708.
Vardhini, B. V., and Anjum, N. A. 2015. Brassinosteroids make plant life easier under abiotic stresses mainly by modulating major components of antioxidant defense system. Environ. Exp. Bot. 51:93-101.

Wang, S. Y., Chen, C. T., and Yin, J. J. 2010. Effect of allyl isothiocyanate on antioxidants and fruit decay of blueberries. Food Chem. 120:199-204.

Wu, H., Liu, X. R., Yu, D. D., Zhang, X., and Feng, J. T. 2014. Effect of ally isothiocyanate on ultra-structure and the activities of four enzymes in adult Sitophilus zeamais. Pestic. Biochem. Physiol. 109:12-17.

Wu, H., Xue, N., Hou, C. L., Feng, J. T., and Zhang, X. 2015. Study on microcapsule preparation of allyl isothiocyanate and its application on mature green tomato preservation. Food Chem. 175:344-349.

Wu, H., Zhang, G. A., Zeng, S. Y., and Lin, K. C. 2009. Extraction of allyl isothiocyanate from horseradish (Armoracia rusticana) and its fumigant insecticidal activity on four stored-product pests of paddy. Pest Manag. Sci. 65:1003-1008.

Wu, H., Zhang, X., Zhang, G. A., Zeng, S. Y., and Lin, K. C. 2011. Antifungal vapour-phase activity of a combination of allyl isothiocyanate and ethyl isothiocyanate against Botrytis cinerea and Penicillium expansum infection on apples. J. Phytopathol. 159:450-455.

Zhou, C. H., Liu, H. X., and Wei, J. 2002. The role of reactive oxygen species in ripening and senescing of fruit. J. Shanghai Jiaotong Univ. 19:77-84 (Agricultural Science). 